\title{
A conceptual framework and a suite of tools to support crisis management
}

\author{
Frederick Benaben \\ Audrey Fertier \\ Aurelie Montarnal \\ Nicolas Salatge \\ Sebastien Truptil \\ Matthieu Lauras \\ Mines Albi - University of Toulouse - FRANCE \\ Sebastien Rebiere \\ firstname.lastname@mines-albi.fr
}

\begin{abstract}
This article aims at describing an approach to support crisis management. The main idea is to use an original vision of Big-Data to manage the question of collaboration issues in crisis response. On the one hand, this article introduces a general framework that structures the methodology applied in our approach. This framework includes several technical and business dimensions and embeds scientific results that are presented in this article or have been described in previous articles. On the other hand, the resulting implemented suite of tools is also presented with regards to the conceptual framework. Finally, in order to emphasize all the main features described in this article, both the framework and the suite of tools are illustrated and put into action through a scenario extracted from a real exercise.
\end{abstract}

\section{Introduction}

Crisis management is the set of activities dedicated to perform: prevention, preparation, response and recovery [1]. Prevention includes the actions dedicated to decrease the probability of risk occurrence. Preparation defines contingency plans to execute in case of occurrence of a crisis. Response concerns the on-the-fly deployment of the defined plans conjunctly with the required adaptation and improvisation activities. Recovery includes tasks and activities dedicated to return to a long-term viable situation. The research works presented in this article mainly focus on the preparation and response phases and aim at providing crisis manager with an agile crisis management approach, equipped with dedicated software components.

This approach inherits from two main statements:

1. On an organizational point of view, operational partners of crisis management may be considered as skilled enough to perform their own missions. However, the main issue concerns the coordination and the collaboration of the responders (who should do what, and when) to avoid any collision, overlap or conflict [2].
2. On a technological point of view, today's world is numeric and the data deluge is a tangible observation in crisis management context as well. Consequently, the question of Big-Data in the specific context of crisis management has to be considered as one of the main avenue [3].

As a consequence and regarding the main objective described above (bold text), the main stake is to succeed in defining an approach able to provide and combine solutions to integrate both these statements: managing the collaboration and dealing with the very huge amount of available data.

The results presented in this article have been obtained in the frame of some types of crisis situations. Actually, the context target is the institutional type of crisis in developed countries. These crises are managed at a local level by dedicated authorities (it may be at a national level at maximum) and imply identified actors (there is no reinforcement by unknown NGO or foreign responders). Flooding of major River in France, industrial accident in Germany, massive transportation accident in Spain are examples of such crises.

The research results presented in this article may be considered from two perspectives: on the one hand, there is a theoretical approach dedicated to support the management of crisis situations. On the other hand, there is also a software suite specifically created and implemented to support that approach.

The approach focuses on the preparation and response phases. It is based on three steps to provide the best response mechanism:

- Define: to design the appropriate response schema according to data, information and knowledge collected during preparation and early response.

- Realize: to actually implement the response schema from the design step.

- Maintain: to adapt and update on-the-fly the response schema according to the evolution of (i) the crisis situation and (ii) the actual perception of the crisis situation (more and more precise).

The software suite (IO-Suite for Interoperability of Organization software suite) is composed of four tools:

- IO-DA (for Design Assistant) aims to support (during the preparation and response phases) the situation modeling and the deduction of the 
response plan (as a collaborative process model). There are three models to be designed: (i) context model describing the stakes (good, people, etc.) and the characteristics of the impacted part of the world, (ii) partner model describing the responders and their capabilities, and (iii) objective model describing the missions to be achieved (i.e. the issues to treat and the risks to prevent). Based on these models, deduction rules (business rules extracted from doctrines and actual plans) are used to infer, from the information embedded in the three models, which capabilities should be used from which partner and in which order to deal with the situation. The result is a BPMN collaborative process model specifically adapted to the current models (i.e. the current knowledge about the situation).

- IO-WA (for workflow assistant) is dedicated to orchestrate the obtained collaborative process. It is based on a workflow engine. When tasks are human tasks, IO-WA uses interfaces that can be deployed on various kinds of devices (smartphone, tablet, computers) to trigger these tasks and get their acknowledgement. When tasks are software tasks, IO-WA uses its service-oriented architecture to run the corresponding services.

- IO-TA (for tracking assistant) is dedicated to monitor both the crisis situation (is there any new event, any new information) and the workflow progress (which task has been done) to constantly control if there is any divergence between the expected situation and the real situation. If so, then IO-TA starts the process again with actual versions of the situation models.

- $\quad$ IO-GA (for governance assistant) is dedicated to manage the models and the associated knowledge base. This is a hidden tool used by the three others. Both these perspectives (approach and tools) can be considered through the following picture.

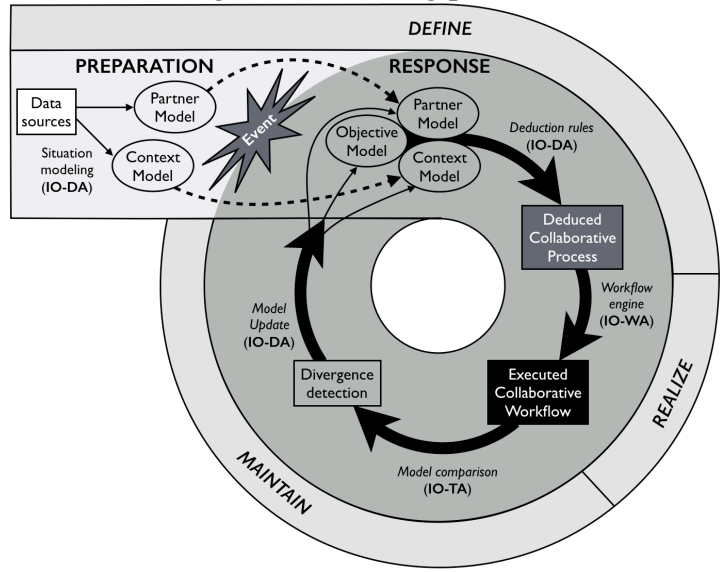

Figure 1. Theoretical framework of the approach.
Considering the specific target context of these research works (local institutional crisis in developed countries), the partner and context models are designed (at least partially) during the preparation phase. Actually the observed administrative / geographic area can be modeled in advance (by listing buildings, populations and various known stakes) in the context model. Similarly, available responders may be modeled in the partner model during the preparation phase (by identifying institutional partners and their resources).

One last element about this approach is that it is an incremental vision of crisis management support: even if the knowledge about the crisis situation is partial, based on the current version of the three models (context model and partner model are supposed to be quite complete), the system can start to deduce and run response collaborative process even if not perfect. The agility mechanism (based on divergence detection) allows adjusting the collaborative process.

This article inherits from [4] and presents updates and progresses. The paper is structured as follows: Section 2 is dedicated to the approach as a whole and to describe the associated conceptual framework. Section 3 presents a literature review and some related works. Section 4 provides details about the proposed approach and the associated tools by introducing a simple use-case, described following the structure of the framework. Finally, a conclusion presents perspectives and limits.

\section{The approach}

This section aims at presenting the defined general approach to reach the objective described in the introduction (bold text) by considering both the statements identified in the previous section. Three steps will be used to present this approach: (i) analysis of the first statement, (ii) analysis of the second statement, and (iii) merging of the results of these analyses.

\subsection{Analysis of the organizational observation}

Managing the collaboration of heterogeneous partners, inheriting from very different cultural backgrounds and very different business capabilities may be achieved by using a collaborative business process approach [5]. In such a case, there are obviously three main tasks to be considered:

- Define: defining the collaboration schema(s) is mandatory. This task is in charge of designing the coordination model(s) and to describe who should do what and when. 
- Realize: realizing the defined collaboration is absolutely necessary. Actually, schema(s) must be orchestrated or choreographed to be useful. The simple definition of the collaboration schema(s) cannot be considered as a final result. Plans, even if well described and relevant, will not be considered in real-time if requiring active involvement of stakeholders (due to the stress, emergency feelings and the time-consuming aspect of plans' reading).

- Maintain: maintaining the collaboration schema(s) is mandatory as well, especially in crisis management context. By definition, crises are unstable situations: there may be additional crisis, unexpected consequences and aftershocks. Consequently, providing the crisis managers with an approach dedicated to support collaborative behaviors also requires features for agility.

These three main components of crisis management collaboration are definitely in line with the steps of the life-cycle of collaborative situations of organizations as described in [6].

\subsection{Analysis of the technological observation}

Exploiting and using relevantly data flows in the frame of crisis management is a critically arduous challenge. The obvious vision of this Big-Data question concerns the following two main requirements: collecting data and exploiting gathered data. However, as described in [7], the domain of crisis management is strictly different from usual domain where Big-Data is used:

- In crisis management the data source world is an open space. Crisis management domain is completely different from production chain or business intelligence, where data sources are mainly well known, usually trustable and cover a clearly defined geographical perimeter. In crisis management, sensors, social networks, opendata and other data sources are initially undefined, potentially non-dedicated and not trustable.

- In crisis management, the exploitation world is an open space as well. Similarly, while in production chain or business intelligence, objectives fixed to the exploitation of the gathered data are well known and defined by the Big-Data user(s) on purpose, in crisis management context, these objectives fixed to the Big-Data treatment are massively unknown preliminarily: what will be the risks to prevent, will there be a fire to fight, an explosion to prevent or the decision of an evacuation to take?

Consequently, according to [7] and [8], in crisis management domain, one available vision is to include an interpretation layer between the data gathering layer and the exploitation layer. The abstraction layers to be considered to manage data and benefit from the data deluge are the following:

- Data level is dedicated to the five following main functions: (i) data source discovery to find available data sources on the considered geographical area, (ii) understanding to reduce the data sources to the one providing data that may be useful for the crisis management, (iii) trust analysis to filter only relevant data (i.e. providing relevant content and trustable), (iv) gathering to collect and assemble the remaining data, and (v) storage to structure the capitalized data repository. This level is in charge of managing the openness of the data source world.

- Information level is dedicated to the interpretation of the collected data. This is the layer in charge of combining, merging and contextualizing the gathered data. The question of syntactic and semantic interpretation, as well as sensemaking [9] is at the heart of this layer. The main objective of this level is to obtain situation models automatically built form the gathered data. Consequently, the mechanism is to instantiate models respecting metamodels, based on the gathered data.

- Action level is dedicated to perform the exploitation of models that have been defined at the information level. All results inherit directly from Model-Driven Engineering (MDE) [10]. A large scale of exploitation schemas may be applied at this level on the obtained models, depending on the goal of the approach.

This three-level structure is a relevant manner to specialize and extend the Big-Data classical framework to fit the "open" field of crisis management.

\subsection{Cross considerations}

To describe the conceptual framework of these research results, this article crosses the results of the two previous subsections to build a two-dimensional framework. Actually, the main goal of the whole approach is then to perform the three steps of the collaboration life-cycle (define, realize and maintain) by moving across the three abstraction layers of data management (data, information and action):

- Define: data must be gathered (data level), then used to build situation model(s) (information level), which will be exploited to deduce relevant collaborative process(es) (action level).

- Realize: the obtained model(s) (at action level) must be evaluated and validated (at action level) to 
be deployed (as workflow models at information level) before being orchestrated or choreographed (at data level).

- Maintain: the orchestrated workflows must be monitored (at data level) to update the situation model(s) (at information level), and detect any disruption or unexpected state to suggest relevant adaptation measures (at action level).

By considering the three steps (define, realize and maintain) as the "life-cycle dimension" and the three layers (data, information, action) as the "abstraction level", it is possible to define a two-dimensional framework. Besides, within this 2D framework, it is also possible to design the journey represented by the whole approach described in this article: The first step is about data gathering to build situation models and deduce collaborative process models. So, it raises from data (gathering) to information (modeling) and then to action (deducing) during the define stage. Then, it is about workflow evaluation, deployment and orchestration. Hence, it dives from action (evaluation), to information (deployment) and then to data (orchestration) during the realize stage. Finally, it is again about raising from data (monitoring), to information (updating models) and then to action (detection and adaptation) in the maintain stage.

The following picture illustrates this whole journey in the two-dimensional framework and also presents the IO-Suite tools within this framework:

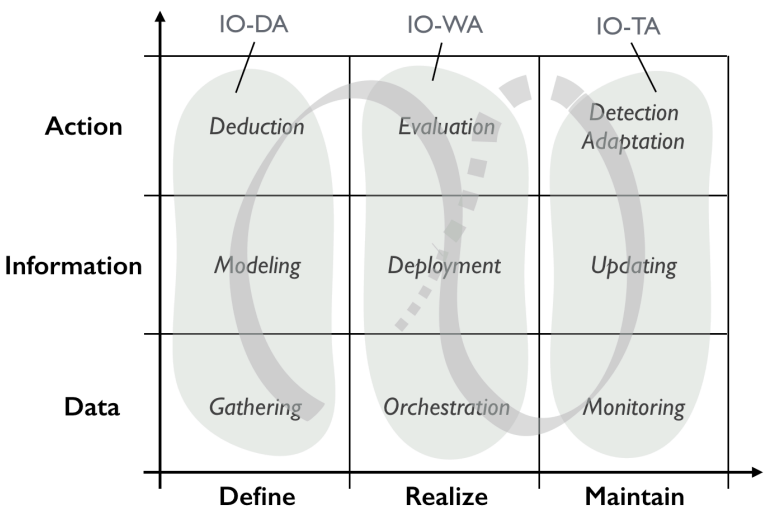

Figure 2. Two dimension framework and IO-Suite.

However, the required underlying knowledge management is not considered in the previous twodimensional framework. Behind each of the nine cells, there are elements about tools and rules to manage instances or concepts. For instance, to build models from the collected data, it may be necessary to compare the data with existing instances in the knowledge base and then to instantiate concepts, according to the results of this comparison to create the actual model. Actually, the idea is very anthropomorphic: if one gives someone a Rubik's
cubeC (assuming he never saw one), then, this person may observe it (touch, look, taste, listen... i.e. data gathering) compare it to his own memories (it looks like Lego(C) and is colored like Mastermind(C... i.e. instances) and create a new instance (this is probably a new toy or a new puzzle... i.e. new instance of concept of the metamodel).

Based, on these considerations, a third dimension may be added to the previous framework to represent the knowledge management dimension. Consequently, the journey presented on figure 2 may actually be considered as the front face projection of a threedimensional journey. The last tool (IO-GA) is specifically dedicated to implement this third dimension. The following picture illustrate this framework:

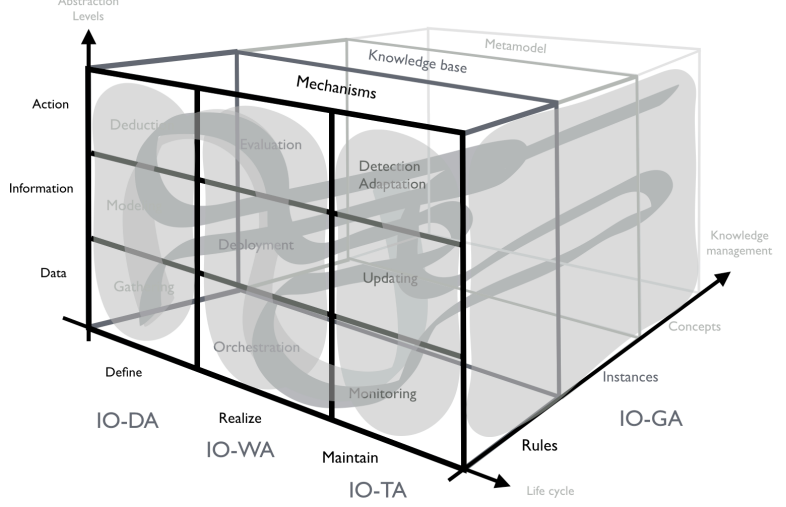

Figure 3. Three dimension framework and IO-Suite.

The objective now is to provide tools that will support the crisis manager to travel all along the path, in this framework, in order to perform an efficient and relevant crisis management.

\section{Related works}

Related works are presented according to the three dimensions of Figure 3: (i) Big-Data (Abstraction level dimension), (ii) Collaboration (Life cycle) and (iii) metamodel (knowledge management).

\subsection{Big-Data}

According to [11] and [12], Big-Data can be considered as a process that facilitates the decisionmaking, through a swift analysis of large amounts of data, of different types, from a variety of sources, to produce a stream of actionable knowledge.

Very classically, Big-Data is considered according to four main directions, also known as the four "V":

- Volume refers to the very large amount of data generated per time unit. Data is gathered and stored to be analyzed, [13] and [14]. 
- Variety refers to the wide diversity of data types (numbers, text, videos, pictures, sounds...) from both known and unknown sources [15] and [16].

- Velocity refers to the frequency of data production and the time required to process the data [12], [13] and [14].

- Veracity refers to trust, objectivity, authenticity, and security surrounding data [15] and [16].

Current academic and industrial researches provide tools [17] that mainly include solutions for at least one of these features. For instance, volume can be tackled by Map Reduce (an approach dedicated to process very large amount): querying the gathered data through that kind of tool would help to reduce the volume of remaining relevant data [18]. Similarly, variety can be managed thanks to the existence of metadata, which allow the identification of the data content [15]. However, there is no single solution that takes into account veracity [20]: Consequently, objectivity, truthfulness and credibility of data need to be controlled, especially in a crisis context.

\subsection{Collaboration}

Over the recent years a considerable number of methods and tools have been developed for collaborative networks (CNs) (e.g. dynamic consortia management, trust management, value systems, incentive mechanisms, etc.). However, most of these developments in CNs focus on networks of enterprises [21]. As such, whilst current collaboration models and tools have potential, they are not fully suited to the specific requirements of a collaborative environment in crisis management that needs some adaptations [4]. One interesting aspect of managing networks in crisis environment is that it is necessary to facilitate collaborations between formal and informal organizational structures. A particular attention needs to be devoted to the elaboration of proper incentive mechanisms, aligned with the value systems of the various stakeholders. On the other hand, new coordination mechanisms need to be devised to cope with emergency situations, incomplete information, and potential unavailability of resources (when parts of the infrastructure are affected by a disaster). Adequate governance mechanisms taking into account the specific requirements, which are different from business contexts, should also be considered.

\subsection{Metamodel}

The crisis management metamodel (presented in [4]) is structured according to a core metamodel and four packages covering Context, Partners, Objectives and Behavior).

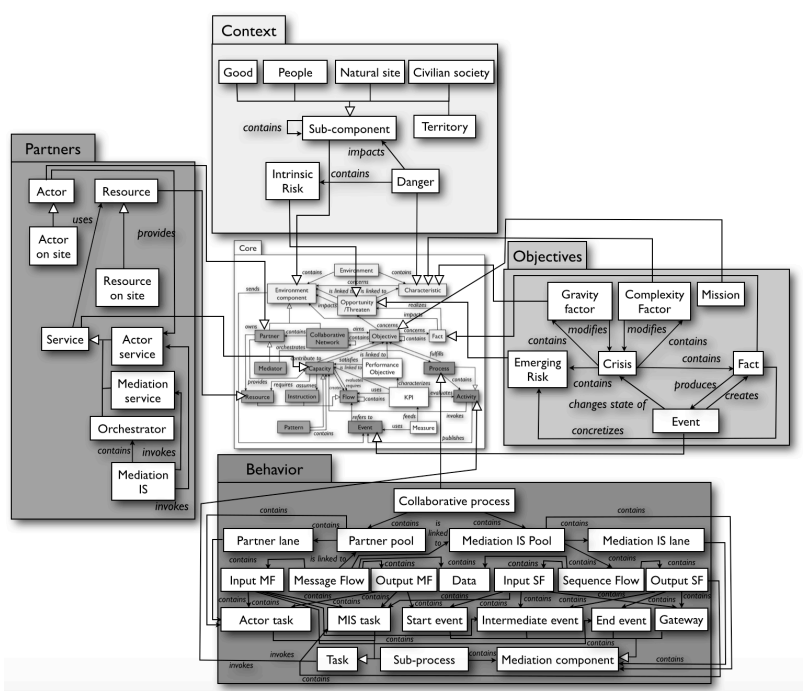

Figure 4. Crisis management metamodel (from [4])

Some more explanation can be given on the concepts included in this metamodel:

Context package:

- Good: human-made elements (building).

- People: group of person (students).

- Natural site: natural element (lake).

- Civilian society: social actors (media).

- Territory: administrative area (county)

- Danger: dangerous feature (seismic area).

- Intrinsic risk: permanent risk (earthquake).

Partners package:

- Actor: stakeholder (firemen).

- Resource: resource used by actors (truck).

- Service: capability of actors (evacuate).

- Actor service: service provided by actors.

- Mediation service: Mediation IS service.

Objectives package.

- Emerging risk: risk arising (panic).

- Effect: direct consequence (fire).

- Mission: objective covering risk or effect.

- Event: event during crisis.

- Gravity factor: characteristic of the crisis that may change its gravity.

- Complexity factor: characteristic of the crisis that may change its type.

The fourth package (behavior) describes concepts from business process. Finally, the aim is to build models of Context, Partners and Objectives in order to use model transformation to obtain Behavior models.

\section{Proposal}

To describe the IO-Suite tools the following usecase will be considered: 
Students of a University are doing sport into the gym. Some students find a white powder in the changing-rooms and start feeling sick (respiratory problems). They call emergency services. When the Firemen arrive on site, due to the complexity of the situation, associated emerging risks and so on, the crisis situation is declared and the local administrative unit (Prefecture) is in charge of chairing the crisis cell. This crisis cell involves Firemen, Police, Red-Cross and Emergency Medical Services.

In the following subsections, the IO-Suite tools are presented from the user point of view. As described in the introduction section, there are four main tools in IO-Suite. Besides, the journey presented on Figure 1 is used to structure the description of IO-Suite, column by column. The third dimension of knowledge management is mentioned in each cell where it is necessary.

\subsection{Define column}

The first stage of the approach is to collect data from the crisis situation in order to build models (covering context, partners and objectives points of view). As explained in introduction, context and partner models are defined during the preparation phase. To illustrate this use-case, only the impacted subparts of these models are illustrated. The following pictures (figures 5,6 and 7) present these models.

The Context model includes the university, the gym, the parking (instances of good concept) and inside the gym there are sport players (instance of people concept) while inside the university there are students, teachers and visitors (instance of people concept).

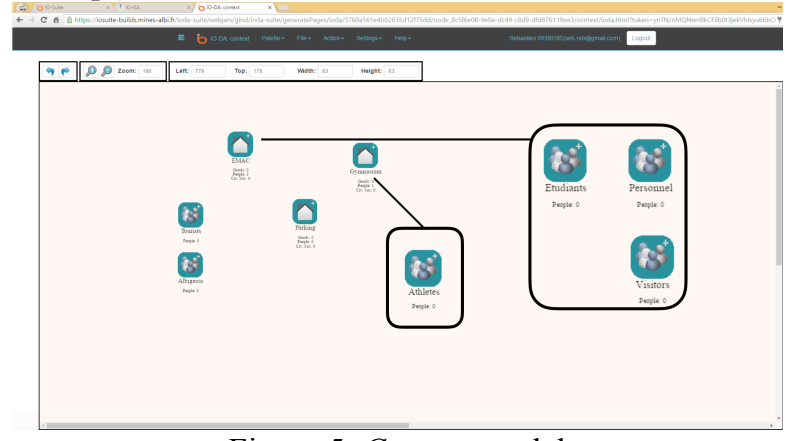

Figure 5. Context model

The Partners model describes the four responders Firefighters, EMS, Red Cross and Police (instance of actor concept) and their respective capabilities such as evacuate victims, identify severely contaminated victims, decontaminate victims, establish security perimeter (instance of service concept).

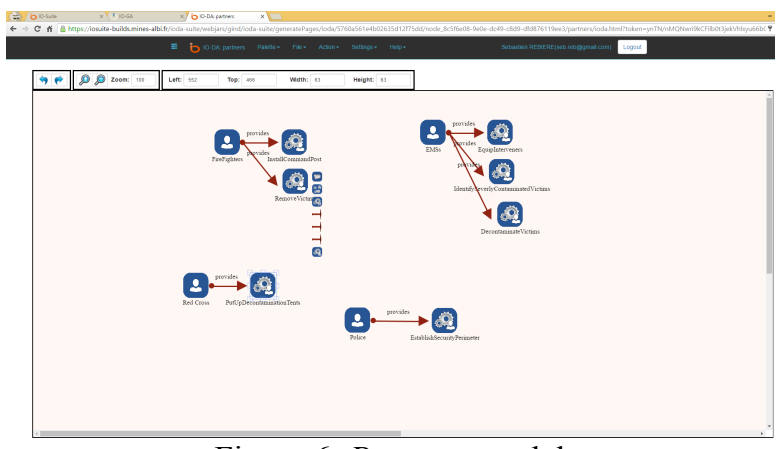

Figure 6. Partners model

The Objectives model is defined during the response phase (as soon as possible) and is based on the available data. It mainly describes all identified risks and issues, and associates one mission to each of them (we believe that there is no objective that is not the result of risk prevention or effect treatment). In the considered use-case, there are instances of the risk concept (outside contamination, panic) and instances of the effect concept (contaminated people).

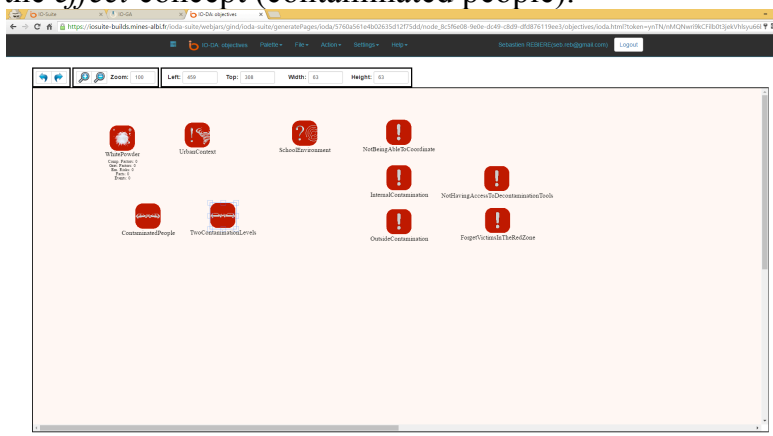

Figure 7. Objectives models

These three models are stored in graph databases (see figure 8), which embeds the knowledge stored in the IO-GA tool (structured according to the metamodel of figure 4). This knowledge base is used intensively by the deduction rules. Actually, all newly created instances are compared with those already included in the knowledge graph to establish semantic proximity relationships. These relationships are used by the deduction rules (define) and detection rules (maintain).

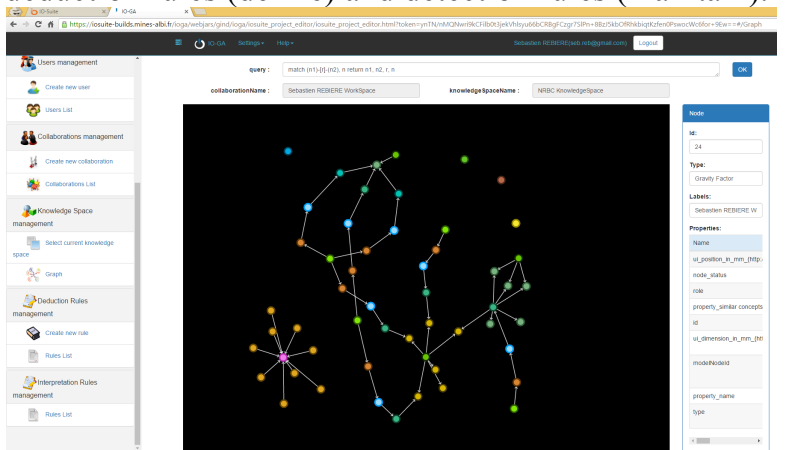

Figure 8. 3D Graph representing the created models 
Then, to move to the upper cell of the "define" column, the user may choose one of the implemented deduction strategies to automatically deduce, from the previous models, the collaborative business process that is supposed to be appropriate for the considered crisis situation. There are actually three available deduction strategies: one is based on an ant colony algorithm, the second one is based on a $0+$ order logic and finally, the third one is based on a multi-attribute approach (MAUT) and Choquet integral (see figure 9). By selecting and applying one of the available strategy chosen the user, the system infers a collaborative business process model (figure 10).

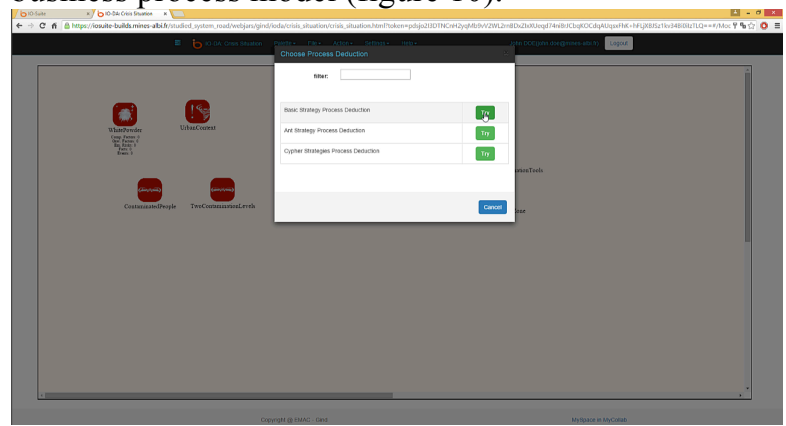

Figure 9. Strategies for business process deduction

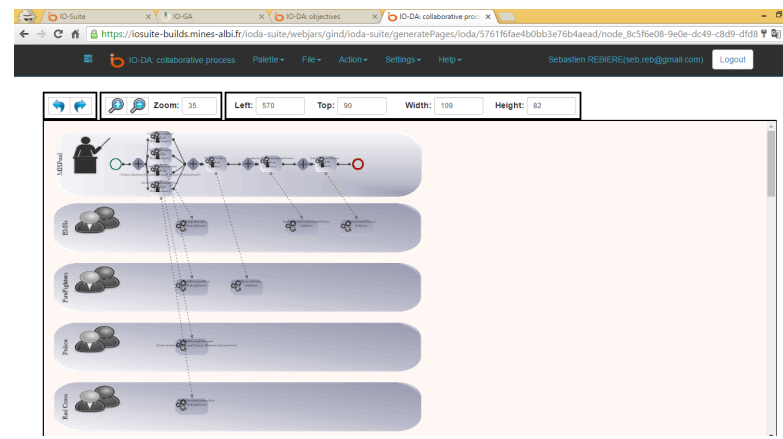

Figure 10. Part of the deduced collaborative process

At the current stage, there is no rational dedicated service to select the appropriate strategy. The user is free to choose. However, there are current research works dedicated to massively evaluate these strategies and define their usage profile. This should help to define, on the one hand, rational and selection criteria, and, on the other hand, potential combination schemas.

The "Define" column is covered by the IO-DA tool (DA for "Design Assistant") and moves from data gathering to collaborative business process design.

\subsection{Realize column}

Unlike the first column ("Define" column), which embeds a bottom-up approach, the second column is top-down. Actually, it starts at the "Action" level with the collaborative business process model that has been deduced on top of "define" column. Then, evaluation and validation (including potential modification) are performed manually by the user directly on the modeling tool.

Once the collaborative schema validated, it can be executed as collaborative workflow (using activiti engine) as presented on figure 11 .

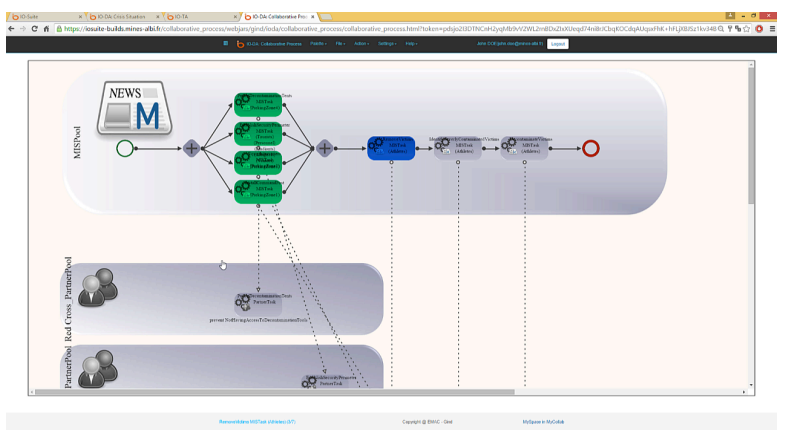

Figure 11. IO-Suite environment

For each activated task, the concerned partner is invoked through any of his connected device. The invocation is a pop-up window asking for the execution of expected task. It is critical to notice here that tasks must be at the appropriate granularity level. It actually means that tasks mustn't be too precise (because it would cause interferences with the internal processes of the partners) and mustn't be too vague (because it wouldn't be relevant for partners). Consequently, tasks are defined in IO-Suite according to the same granularity as capabilities described in the Partner model (Figure 6).

The realize column is finally a dive into computerization to move from a formalized collaborative behavior to its concrete orchestration.

\subsection{Maintain column}

The "maintain" column is a bottom-up column. During the orchestration of the process, and as explained in [4], there is a double monitoring: the objective model is duplicated (see figure 12).

One of the model (expected model) is automatically updated based on events coming from the workflow engine (activiti), assuming that if one task is finished, then the objective that was associated is achieved. Consequently, risk(s) may be deleted, resource(s) may be added or modified, etc. The second model (field model), is updated based on actual events coming from the crisis situation and interpreted to create, modify or delete instances on the field model. This mechanism is fully described in [23]. 


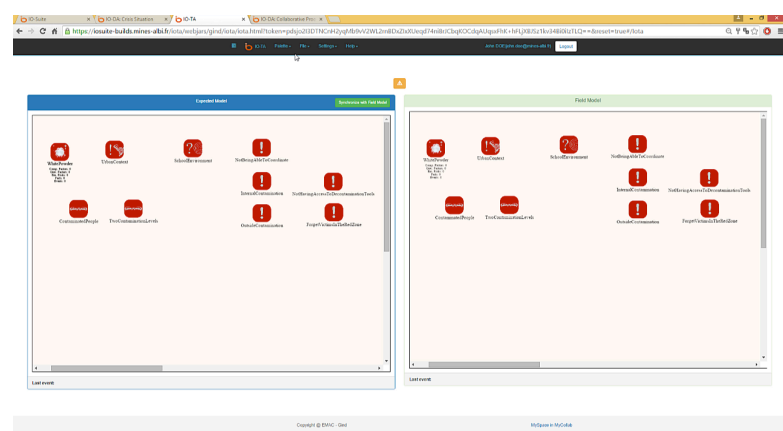

Figure 12. Agility dashboard

As a consequence, these models (expected model and field model) may diverge. The system is then able to detect this difference and to evaluate the distance between both these models (based on weight of concepts). The following picture presents both these models once they have been diverging.

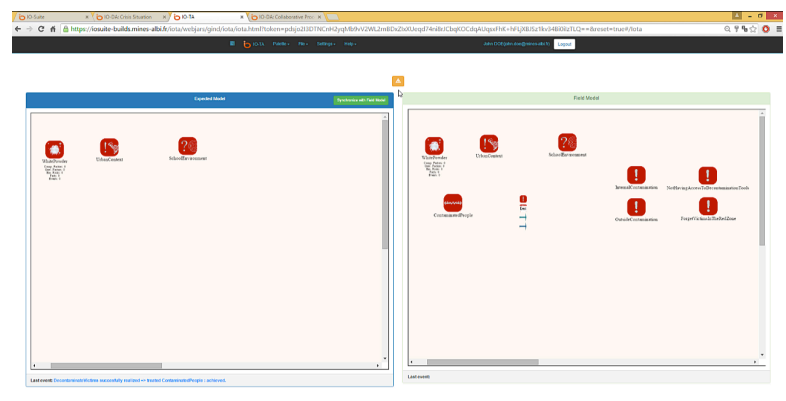

Figure 13. Distance between the monitoring models

If the distance is over a threshold, then an alert is generated and the workflow orchestration is interrupted to adapt the collaborative behavior. Actually, this twosteps mechanism (detection and adaptation) is based on the definition of agility extracted from [23]: agility = (detection + adaptation) $x$ (effectiveness + reactivity).

As a consequence, the system is able to use one of the available deduction strategies to build the new model of the behavior (just as it is done on top of the "define" column) as presented on figure 14 .

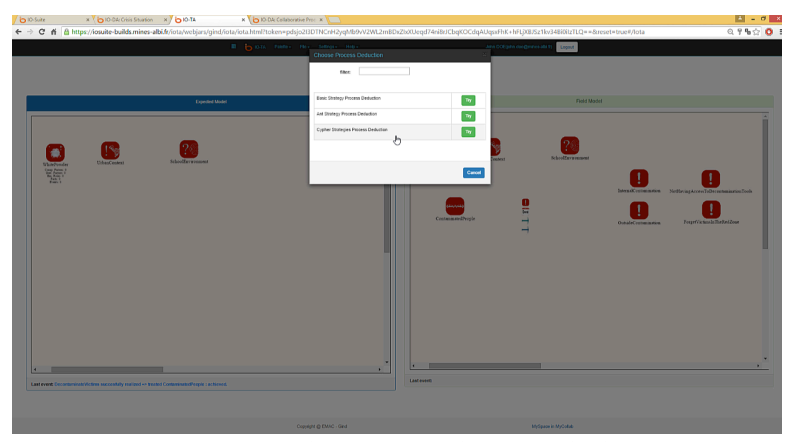

Figure 14. Deduction of the next behavior
On a more theoretical point of view, this adaptation is based on the analysis of the distance between both the models (expected and field models). As far as this measure does not concern the distance between two points, but between two clouds of points, it is possible to get a rich characterization of this distance. As presented in [23], this rich characterization allows to choose between a "large" adaptation (deduction of a new process because the difference between models shows a deep modification of the situation), a "medium" adaptation (modification of the business process model because the difference between models shows a change into the partner network and their capabilities), or a "small" adaptation (re-execution of the workflow because the business process and the network of partners remain relevant but there have been dysfunctions in the orchestration).

\section{Conclusion}

In this article the results presented in [4] have been extended and improved. The first result is a very theoretical one and concerns the three-dimensional framework that can be used to define trajectories and journeys. The second result is a very practical one and concerns the software suite (IO-suite) that has been implemented to support this approach. IO-suite is composed with four main tools:

- IO-DA: Design Assistant covering the first column "Define". However, and as presented in [22], this tool does not entirely cover the first column but mainly the two upper thirds. The lower third is manually supported.

- IO-WA: Workflow Assistant covering the second column "Realize". Nevertheless, this tool mainly covers the two lower thirds of this column. The evaluation and selection of the process model is still a human task. However, there are a lot of tools and research results that are dedicated to evaluate business process models. This integration might not be difficult.

- IO-TA: Tracking Assistant covering the third column "Maintain". The current research works on the interpretation of data (presented in [22]) will also contribute to improve the coverage of this tool (currently covering the two upper thirds of the third column). Actually, even if the updating of the expected model is automatized (based on data coming from the orchestration), the updating of the field model is a manual task.

- IO-GA: Governance Assistant covering the third dimension of the three-dimensional framework. This assistant is in charge of managing models, rules, knowledge bases and metamodel. 
The following picture presents the framework and the position of IO-DA, IO-WA, IO-TA and IO-GA into this framework.

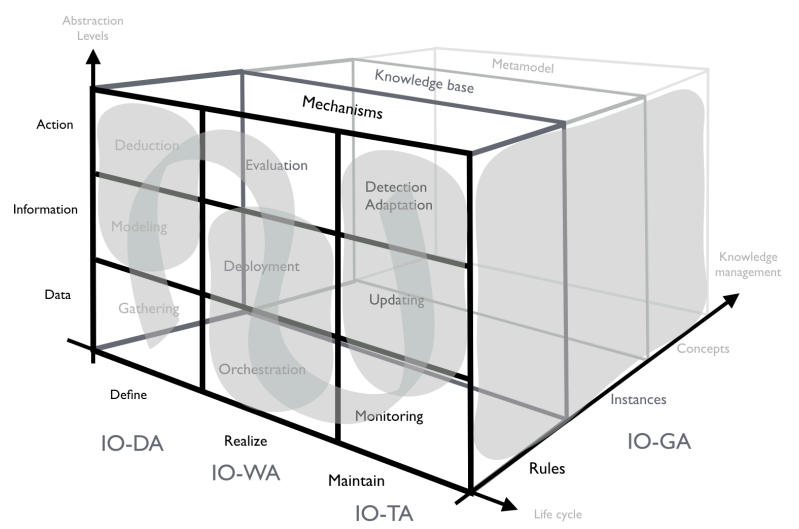

Figure 15. IO-Suite tools on the 3D-Framework

Consequently, considering the current status of IOsuite, the main perspective is about the two following points (in line with the representation of figure 2):

- Improve the coverage of IO-DA, IO-WA and IO-

TA to support the user in the tasks of modeling, evaluation and updating of models. This is a work in progress and two $\mathrm{PhD}$ students are currently working on these subjects within the frame of two funded projects (one National and one European).

- Refine the definition of the trajectory of crisis management in the three-dimensional framework to include concretely the third dimensions and all expectations in terms of knowledge management.

The following picture presents these perspectives and the differences between figure 15 and figure 2 define the avenues of research.

The last short-term improvement to be considered concerns the metamodel and the management of its lifecycle. Currently, it is not managed at all. Improvements, evolutions and changes in the metamodel are pure human tasks. However, regarding the interpretation potential, it is interesting to consider that the unused data (data that are not directly usable to instantiate concepts of the metamodel) may imply the creation of new concepts. For instance, considering the Rubik's cube example described earlier, the person manipulating the Rubik's cube may not know the concepts of puzzle or brain teaser. In that case, he/she may discover the concept by him/her self but he/she may also be taught by someone else this new concept. This is a very anthropomorphic learning process.

Finally, one long-term perspective is about generalization of the considered domain to more demanding crises and catastrophes (by considering [24] and [25]). There are three main obstacles to this generalization:

- The first one concerns modeling time. Context and partner models are supposed to be defined and updated during the preparation phase. In the case of a humanitarian crisis, the available partners and the impacted area cannot be predefined. Consequently, the three models (context, partner and objective) must be defined during the response phase. This is obviously a very strong drawback. However, the current research works on automated data interpretation for model design and update may eventually solve this issue by providing a way to collect data from very heterogeneous data sources (sensors, open data, social networks, GIS, etc.) and instantaneously create/update/delete instances of appropriate concepts.

- The second one concerns the deduction and detection rules. While these rules are currently based on national doctrine and plans, a more open system should be based on "universal" (or configurable) business rules. This is also a very strong drawback.

- The third one concerns the knowledge base. The current knowledge base contains instances from local and national scenarios. This is obviously far from exhaustive in the perspective of a more open system. However, there are existing disaster knowledge bases and ontologies that could be exploited to create a more open knowledge base.

Finally, the results presented in this article are dedicated to a precise type of crisis. However, this type of crisis is not marginal at all. Besides, the presented approach is not only based on theoretical considerations. Not only does this approach rely on a theoretical framework structuring big-data abstraction levels, crisis management life-cycle and knowledge management dimensions, but also it is based on a software suite that implements the whole approach.

\section{References}

[1] N. Atlay, W. Green, "OR/MS research in disaster operations management" European Journal of Operation Research, Elsevier, vol. 175, issue 1, pp. 475-493, 2005.

[2] F. Benaben, S. Truptil, M. Lauras, N. Salatge, "Management of collaborative behavior through a serviceoriented mediation system: the case of crisis management" Proceedings of IEEE International Conference on Service Computing (SCC 15), pp. 554-561, 2015.

[3] P. Meier, "Strengthening humanitarian information: the role of technology", Chapter 3 of World Disaster Report 
2013, International Federation of Red Cross, Red Crescent Societies (IFRC), 2013.

[4] F. Benaben, S. Truptil, M. Lauras, N. Salatge "A Metamodel for Knowledge Management in Crisis Management", Proceedings HICSS 16, IEEE, pp. 126-135, 2016.

[5] T. Gulledge, R. Sommer, "Business process management: public sector implications", Business Process Management Journal, Emerald Insight, vol. 8, issue 4, pp. 364-376. 2002.

[6] L. Camarinha-Matos, H. Afsarmanesh, N. Galeano, A. Molina, "Collaborative networked organizations-Concepts and practice in manufacturing enterprises". Computers \& Industrial Engineering, Pergamon, vol. 57, issue 1, pp. 46-60, 2009.

[7] F. Benaben, A. Montarnal, A. Fertier, S. Truptil "BigData and the question of horizontal and vertical intelligence: a discussion on disaster management", Proceedings of IFIP Working conference on Virtual Enterprise (PRO-VE 16), to be published, Springer, 2016.

[8] F. Benaben, "A Formal Framework for Crisis Management describing information flows and functional structure", Proceedings of Humanitarian Technology conference, to be published, Elsevier, 2016.

[9] W. Muhren, B. Van De Walle, "A Call for sensemaking support systems in crisis management" Chapter in Interactive Collaborative Information System, Springer Berlin Heidelberg, pp. 425-452, 2010.

[10] A. Rodrigues Da Silva "Model-driven engineering: A survey supported by the unified conceptual model" Computer Languages, Systems and Structures, Elsevier, vol. 43, pp. 139-155, 2015.

[11] J. Kalyvas, “A Big Data Primer for Executives” Chapter in Big Data, CRC Press, 2015.

[12] D. Power 'Using 'Big Data' for analytics and decision support" Journal of Decision Systems, Elsevier, vol. 23, issue 2, p. 222-228, 2014.

[13] Y. Demchenko, P. Grosso, C. De Laat, "Addressing big data issues in scientific data infrastructure" Proceedings of Collaboration Technologies and Systems (CTS 13), IEEE World Congress, pp. 48-55.
[14] I. Hashem, I. Yaqoob, N. Anuar, "The rise of Big Data on cloud computing: Review and open research issues" Information Systems, Pergamon, vol. 47, pp. 98-115, 2015.

[15] K. Krishnan K. "Data Warehousing in the age of big data” Elsevier Science \& Technology Books, 2013.

[16] F. Ohlhorst "Big data analytics: turning big data into big money” John Wiley \& Sons, 2012.

[17] S. Chalmers, C. Bothorel, R. Clemente, "Big Data-State of the Art”. Internal report Telecom Bretagne, 2013.

[18] K. Grolinger, M. Hayes, W. Higashino "Challenges for mapreduce in big data" Proceedings of Services (SERVICES), IEEE World Congress, pp. 182-189, 2014.

[19] S. Kaisler, F. Armour, J. Espinosa " Big data: Issues and challenges moving forward" Proceedings of Hawaii International Conference on System Sciences (HICSS 13), IEEE, pp. 995-1004, 2013.

[20] T. Lukoianova, V. Rubin "Veracity roadmap: Is big data objective, truthful and credible?" Advances in Classification Research Online, vol. 24, issue 1, pp. 4-15, 2014.

[21] L. Camarinha-Matos, H. Afsarmanesh, "Collaborative Networks: a new scientific discipline" Journal of Intelligent Manufacturing, Kluwer Academics Publishers, vol. 16, issue 4-5, pp. 439-452, 2005.

[22] A. Fertier, A. Montarnal, A.-M. Barthe-Delanoe, S. Truptil, F. Benaben, "Adoption of big data in crisis management toward a better support in decision-making" Proceedings of Conference on Information System for Crisis Response And Management (ISCRAM 16). 2016.

[23] A.-M. Barthe, S. Truptil, F. Benaben, H. Pingaud "Event-Driven agility of interoperability during the run-time of collaborative processes", Decision Support Systems, Elsevier, vol. 59, pp. 171-179, 2014.

[24] N. Simpson, P. Hancock "Fifty Years of Operational Research and Emergency Response", Journal of the Operational Research Society, Springer, vol. 60, pp. S126S139, 2009.

[25] J.J. Gonzalez, L. Labaka, S. Hiltz, M. Turrof "Insights from a Simulation Model of Disaster Response: Generalization and Action Points". Proceedings HICSS 15, IEEE, pp. 152-161, 2015. 